\title{
FAMILY OFFICE: ESTRUTURA ORGANIZATIVA DE GESTÃO DE RIQUEZA FAMILIAR
}

\author{
Caroline de Oliveira Orth ${ }^{1}$, Melissa Gerhard ${ }^{2}$, Carine Oliveira ${ }^{2}$, Clea Beatriz Macagnan ${ }^{2}$ \\ Universidade Federal do Rio Grande do Sul- UFRGS ${ }^{1}$, Universidade do Vale do Rio dos Sinos - UNISINOS ${ }^{2}$ \\ caroline.ortheufrgs.br, mgerhard@unisinos.br, carine@unbisinos.br, \\ cleabeatrizmegmail.com
}

\section{Resumo}

O presente artigo analisou publicações sobre o tema family offices (estruturas organizativas que administram a riqueza da família e prestam todo tipo de serviços para as mesmas). Esta estrutura organizativa pode ser utilizada como parte do plano de governança familiar. Family offices surgiram, inicialmente, para auxiliar as famílias no gerenciamento do produto gerado pelo negócio: a riqueza. Atualmente, os family offices podem prestar todo o tipo de serviço profissional. Os resultados encontrados mediante a análise realizada permitem concluir que há pouca pesquisa científica sobre family office, tanto nacional, quanto internacionalmente. Analisou-se uma amostra de 37 (trinta e sete) artigos, dos quais, 32 (trinta e dois) são de autoria internacional e 5 (cinco) são de autoria nacional. Os artigos foram escritos no período de 2001 a 2011. Há um predomínio de trabalhos opinativos (sem apresentar rigor científico) abrangendo $70 \%$ (setenta por cento) da amostra. Apenas 5\% (cinco por cento) apresentam abordagem empírica. Esses resultados demonstram que há uma lacuna a ser preenchida com pesquisas empíricas sobre family office tanto no Brasil, quanto no exterior.

Palavras-chave: Gamily Office. Governança Familiar.

\section{FAMILY OFFICE: ORGANIZATIONAL STRUCTURE OF HOUSEHOLD WEALTH MANAGEMENT}

\begin{abstract}
This paper reviewed publications about the family offices subject (organizational structures that manage the family wealth and provide all kinds of services for these families). This organizational structure can be used as part of the family governance plan. Family offices appeared initially to assist families in managing the product generated by the business: wealth. Currently, family offices can provide all kind of professional service. The results found by the analysis indicate that there are few scientific researches on family office, both international and nationwide. We analyzed a sample of 37 (thirty-seven) articles, of which 32 (thirty two) of them are international and five (5) are national authorship. The articles were written from 2001 to 2011. There is a predominance of opinionative researches (no scientific rigor) covering $70 \%$ (seventy percent) of the sample. Only 5\% (five percent) presents a empirical approach. These results demonstrate that there is a gap to be filled with empirical research on family office both in Brazil and abroad.
\end{abstract}

Keywords: Family Office. Family Governance.

\section{Introdução}

O presente artigo analisou publicações sobre o tema family offices (estruturas profissionais que prestam todo tipo de serviços para famílias ricas). O estudo foi realizado mediante uma revisão de literatura de publicações nos principais periódicos com projeção nacional ou internacional, o que permitiu identificar e selecionar os trabalhos sobre family offices. Essa seleção poderá ser utilizada como referencial teórico em trabalhos sobre o tema no Brasil, contribuindo assim para 
futuras pesquisas nessa área que começa a oferecer um novo mercado de atuação para os contadores, consultores financeiros, advogados e auditores.

A partir da pesquisa efetuada foi possível perceber que não há um conceito para family office, tampouco um consenso quanto a sua forma de atuação e estrutura. Carrol (2001), descreve os serviços prestados pelo family office como "serviços personalizados" e Maslinski (2006) entende que essa estrutura pode ser projetada para prestar qualquer tipo de serviço que atenda aos interesses de uma família. Embora não exista consenso quanto ao conceito e a estrutura de um family office, diversos autores, tais como Carrol (2001), Garnham (2001), Heinz (2003), Montford (2004), Gray (2005) e Maslinski (2006) apontam o family office como a solução para o atendimento das necessidades das famílias ricas. Alguns autores ainda abordam a atuação do family office como importante instrumento de governança corporativa Maslinski (2006), Isdale (2006) e (Gray 2007).

Os estudos encontrados a nível nacional não abordam exatamente o tema family office, e quando abordam o fazem com superficialidade. Os autores brasileiros focam a empresa familiar e suas questões de governança, como, por exemplo, o trabalho de Escuder (2005), Mizumoto e Machado Filho (2007) e Dias e Nenevê (2010). O único trabalho específico sobre family office foi o trabalho de conclusão para o curso de pós-graduação em Administração na Universidade Federal do Rio Grande do Sul escrito por Aquino (2009). O objetivo do trabalho, segundo o autor, foi o de definir family office e demonstrar o seu funcionamento no Brasil. O trabalho fundamenta-se em informações coletadas da internet, mas especificamente de sites de instituições que oferecem esse tipo de serviço, bem como de reportagens que abordam o assunto.

Sendo assim, percebe-se a necessidade de ampliar as pesquisas sobre os family offices, tanto a nível nacional quanto internacional. Este artigo esta estruturado, apos esta introdução, com abordagem dos seguintes itens: family offices, governança, metodologia utilizada para o estudo e os meios de levantamento de dados; os resultados e as considerações finais.

\section{Family Office}

Estudos demonstram que a utilização de estruturas de Family office, na prática, já ocorre há vários anos. Gray (2005) aponta que os primeiros grandes empresários americanos, tais como os Rockefellers, Carnegies, Fords, Vanderbilts, Roosevelts, foram os pioneiros em utilizar os modelos de gestão de riqueza através de estruturas centralizadas as quais hoje se denominam de family offices. A necessidade de constituição dessa estrutura centralizada de gestão surgiu a partir da obrigação de gerenciar o subproduto das empresas: a riqueza por elas gerada. Os empresários perceberam que não tinham nem tempo nem conhecimentos para uma gestão bem sucedida. A primeira solução foi entregar a responsabilidade pela gestão de riqueza aos membros do pessoal corporativo interno. Essa decisão acarretou além de sobrecarga do pessoal, em problemas de privacidade (com o conhecimento de assuntos familiares pessoais). Além desses fatores, a riqueza tornou-se cada vez mais exigente o que aumentou a dependência de empresários e investidores ricos em consultoria especializada de profissionais da área financeira. As questões tributárias também contribuíram para o aumento da complexidade da gestão financeira da família. (GRAY, 2005)

Embora se observe a utilização dos family offices já há bastante tempo, não há um conceito definido para os mesmos. Carrol (2001), entende que o family office é uma estrutura que visa a prestação de serviços personalizados. Na mesma linha, Maslinski (2006) entende que essa estrutura pode ser projetada para prestar qualquer tipo de serviço que atenda aos interesses de uma família. Embora, não exista consenso quanto ao conceito e estrutura de um family office, diversos autores, tais como Carrol (2001), Garnham (2001), Heinz (2003), Montford (2004), Gray (2005) e Maslinski (2006) o apontam como a solução para o atendimento das necessidades das famílias ricas. Já Lowenhaupt (2008) critica a gestão compartilhada, ou seja, gestão “em 
família", como defendem os autores anteriores. Ele entende que cada indivíduo deve buscar a gestão de seus ativos, privilegiando a liberdade individual.

Um aspecto importante a ser levado em consideração é a identificação do momento adequado para constituir um family office. Garnham (2001), Montfort (2004) e Gray (2005) apontam que o family office é instituído, em muitos casos, após a venda de uma empresa familiar. Montfort (2004) complementa que nos Estados Unidos, essa estrutura é criada para famílias cuja riqueza ultrapassa \$ 30 milhões. No entanto, Hauser (2004) e Gray (2005) destacam que o que determina a necessidade de uma estrutura de gestão é complexidade da riqueza e não o seu tamanho. Gray (2005) exemplifica que uma família de 10 milhões de dólares pode possuir estruturas de propriedade complexas, pode estar sujeita a diversas formas de tributação e possuir vários irmãos ou primos envolvidos no negócio da família. Já uma família de US \$ 100 milhões pode ter a maior parte dos ativos ligados ao negócio principal, pode ter uma estrutura de propriedade mais simples e menos familiares envolvidos no negócio, mesmo através de gerações. Nesse caso a atuação do family office seria mais indicada para o primeiro caso e não, necessariamente, para o segundo.

Sendo assim, não há uma definição clara de qual seria o momento adequado ou o patamar de ativos ideal para a criação de um family office. O que os autores apontam são circunstâncias que podem indicar a necessidade de consultoria especializada. Gray (2005) aponta que a evolução crescente do mercado financeiro e a consequente onda de combinações de negócios, cria complexas considerações para as famílias envolvidas, especialmente em função de que as mesmas não se sabem o que fazer com o recurso que se tornou disponível. Surgem então as seguintes dúvidas: o dinheiro deve simplesmente ser dividido entre a família? Quanto cada membro deve receber? O dinheiro deve ser investido coletivamente? Como isso afetará o legado do fundador? Caso o investimento tenha sido coletivo, como cada membro da família deve tomar decisões de investimento? O fundador deve continuar a suportar o peso na tomada de decisões até que ele ou ela esteja pronto para passar o bastão para a próxima geração? Todas essas definições aliadas ao crescente número de membros da família, ao aumento das taxas de divórcio ameaçando dispersão de riqueza não planejada e o aumento dos custos dos serviços, são aspectos que poderiam ensejar a contração dos serviços de um family office. Além dessas circunstâncias os autores indicam outras, tais como:

\begin{tabular}{|l|l|}
\hline Autores & Circunstâncias que poderiam ensejar a contratação de um familly office \\
\hline $\begin{array}{l}\text { Garnham (2001) Montford } \\
\text { (2004) Gray (2005), Maslinski } \\
\text { (2006) }\end{array}$ & $\begin{array}{l}\text { Complexidade do mercado financeiro (criação de fundos de Hedge e } \\
\text { derivativos, planos de aposentadoria, opções, privaty equity) e a consequente } \\
\text { dificuldade em encontrar gestão especializada de ativos que privilegie os } \\
\text { interesses do cliente }\end{array}$ \\
\hline Garnham (2001) & $\begin{array}{l}\text { Dificuldade em encontrar especialistas tributários capazes de lidar com } \\
\text { questões complexas advindas de investimentos situados ao redor do mundo }\end{array}$ \\
\hline Garnham (2001), Gray (2006) & Falta de preparo das próximas gerações para assumir os negócios da família \\
\hline Garnham (2001) & $\begin{array}{l}\text { Carência de profissionais aptos a trabalhar com a sofisticação do mercado } \\
\text { financeiro }\end{array}$ \\
\hline Montford (2004) & $\begin{array}{l}\text { Riscos de não obtenção de informações suficientes para a formação da } \\
\text { carteira ótima }\end{array}$ \\
\hline Gray (2005) & Aumento do número de membros na família empresária \\
\hline Gray (2005) Maslinski (2006) & Estruturas de propriedade ramificadas e complexas \\
\hline $\begin{array}{l}\text { Gray (2005), Brunel e Gray } \\
\text { (2005), Gray (2006) }\end{array}$ & $\begin{array}{l}\text { Desconhecimento da estrutura, dinâmica e da governança familiar por parte } \\
\text { dos tomadores de decisão }\end{array}$ \\
\hline Gray (2005) & $\begin{array}{l}\text { Falta de tempo e de habilidade para lidar com o produto gerado pelo negócio } \\
\text { da família: riqueza gerada }\end{array}$ \\
\hline Maslinski (2006) & Processos judiciais envolvendo disputas entre familiares \\
\hline
\end{tabular}

Quadro 1 - circunstâncias que ensejam a contratação de family offices (elaborado pelas autoras) 
Outro aspecto importante a destacar diz respeito aos objetivos a serem alcançados pelos family offices. Gray (2005) destaca que as famílias ricas tradicionais como os Rockefellers, após o período da Grande Depressão, passaram a objetivar a preservação da riqueza para as gerações vindouras. Para atingir esse objetivo, passaram a contar com advogados, contadores e outros conselheiros exclusivos para a família. Esse fato pode ser considerado como o embrião do family Office. Carrol (2001) e Garnham (2001) também informam que a preservação da riqueza através do tempo é o principal objetivo do family office. Heinz (2003) e Gray (2007) apontam que o family office, enquanto estrutura de governança familiar deve auxiliar, em última instância, na busca pela felicidade de cada membro da família. Já para Montford (2004) o family office tem como "pedra angular" a identificação e avaliação de prestadores serviço que sejam capazes de atender a todas as exigências impostas por um mercado dinâmico e moderno. Isso porque entende que a os mercados de investimento global exigem habilidades adicionais para avaliar as oportunidades que devem incluir, além de ações, outros investimentos tais como: antiguidades, metais preciosos, ações privadas e outros setores, incluindo a multiplicidade de estratégias de fundos de hedge. Dessa forma, os objetivos de um family office podem ser assim resumidos:

\begin{tabular}{|l|l|}
\hline Autores & Objetivos do family office \\
\hline Carrol (2001), Garnham (2001) & Manter a riqueza da família através de gerações \\
\hline Heinz (2003) Gray (2007) & Auxiliar no alcance da felicidade da família \\
\hline Montford (2004) & Identificação e avaliação de prestadores de serviço \\
\hline
\end{tabular}

Quadro 2 - Objetivos dos family offices (elaborado pelas autoras)

Como já comentado, Maslinski (2006) entende o family office pode ser projetado para prestar qualquer tipo de serviço que atenda aos interesses de uma família. Através da pesquisa realizada foi possível identificas os seguintes tipos de serviços:

\begin{tabular}{|l|l|}
\hline Autores & $\begin{array}{l}\text { Tipos de serviços prestados pelo family } \\
\text { office }\end{array}$ \\
\hline Carrol (2001), Garnham (2001), Heinz (2003), Isdale (2006) & Planejamento tributário \\
\hline Garnham (2001), Heinz (2003) & Planejamento para Filantropia \\
\hline Garnham (2001) & Planejamento sucessório \\
\hline Garnham (2001), Gray (2005) & Estrutura de riqueza \\
\hline Garnham (2001), Heinz (2003), Maslinski (2006) & Gestão de ativos \\
\hline Isdale (2006), & Planejamento financeiro \\
\hline Garnham (2001) & $\begin{array}{l}\text { Gestão de pessoas (funcionários pessoais, } \\
\text { assessores e especialistas) }\end{array}$ \\
\hline Garnham (2001) & $\begin{array}{l}\text { Formalizar os objetivos e a dura de } \\
\text { governança familiar }\end{array}$ \\
\hline $\begin{array}{l}\text { Garnham (2001), Heinz (2003), Hamilton e Godfrey (2006), } \\
\text { Gray (2007) }\end{array}$ & Treinamento para as próximas gerações \\
\hline Gray (2005) & $\begin{array}{l}\text { Educação continuada para os membros da } \\
\text { família }\end{array}$ \\
\hline Heinz (2003), Isdale (2006), & Promover encontros e reuniões de família \\
\hline Isdale (2006), Kilpatrick, (2007), Franke (2008) & Planejamento imobiliário \\
\hline Isdale (2006), Gray (2005) & Planejamento de férias \\
\hline Isdale (2006), & Avaliação de desempenho \\
\hline Isdale (2006), & Orçamento \\
\hline Maslinski (2006), Gray (2005) & Serviços de concierge \\
\hline Maslinski (2006) & Gestão de processos judiciais \\
\hline
\end{tabular}

Quadro 3 - Tipos de serviços prestados por family offices (elaborado pelas autoras) 


\section{Governança}

Conforme aponta Gillian (2006), a definição de governança corporativa não é universal. Isso ocorre, provavelmente, em função dos aspectos sociais, econômicos e culturais que influenciam a Governança Corporativa ao redor do mundo, evidenciados especialmente pelos trabalhos de La Porta, Silanes e Shleifer (1998) e Mork (2005). Em função disso, há inúmeros enunciados para designar governança corporativa. Na visão de Sheifer e Vishny (1997), o termo designa as maneiras pelas quais os fornecedores de capital asseguram-se de obter retorno sobre seu investimento. Essa necessidade de assegurar-se quanto ao retorno do seu investimento decorre do "conflito de agência", abordado por Jensen e Meckling (1976) e ocorre quando o agente (executivo) abusa de seu poder ou não executa suas funções de acordo com os interesses dos financiadores de capital.

Os estudos de Berle e Means (1932) na década de 30 nos Estados Unidos registram o início do debate sobre esse conflito quando colocam que "a separação entre propriedade e controle produz uma situação na qual os interesses do proprietário e os do executivo podem divergir - e muitas vezes divergem realmente." Berle e Means pesquisaram as conseqüências dessa separação e concluíram que à medida que propriedade se dispersava, aumentava o conflito de interesses existentes entre os detentores da propriedade e os gestores. Em função disso defendiam o direito de voto para todos os acionistas, maior transparência e responsabilização.

Após esses estudos, inúmeros outros abordam o tema governança corporativa. Talvez, a principal motivação seja pelo fato de que não há evidência suficiente para afirmar que a adoção de boas práticas de governança realmente resolve o conflito de agência e efetivamente melhora o valor de mercado da empresa. Esses aspectos, até os dias atuais, são questões empíricas, conforme aborda Healy e Palepu (2000). Mesmo assim diversos países passaram a publicar códigos de boas práticas de Governança Corporativa, sendo o primeiro deles o Relatório Cadbury, que surgiu como resposta aos escândalos registrados no mercado corporativo e financeiro da Inglaterra no final dos anos 1980. Após esse código, a França, o Japão e a Alemanha iniciaram ações prevendo boas práticas de governança corporativa. Em 1999 o grupo de governança corporativa da OECD (formado no ano anterior) divulgou a consolidação dos Principles of Corporate Governance.

No Brasil, o assunto começa a ser discutido em 1995 com a fundação do Instituto Brasileiro de Conselheiros de Administração (IBCA), atual Instituto Brasileiro de Governança Corporativa IBGC. O IBGC tem como meta a busca pela excelência em governança através pesquisa, treinamentos e palestras sobre o assunto e destaca que "a preocupação da Governança Corporativa é criar um conjunto eficiente de mecanismos, tanto de incentivos como de monitoramento, a fim de assegurar que o comportamento dos executivos esteja sempre alinhado com o interesse dos acionistas." Além disso, através do Código de Melhores Práticas de Governança Corporativa, o IBGC define que a "Governança Corporativa é o sistema pelo qual as organizações são dirigidas, monitoradas e incentivadas, envolvendo os relacionamentos entre proprietários, Conselho de Administração, Diretoria e órgãos de controle".

Como pode ser observado, as discussões sobre governança corporativa e o conflito de agência iniciaram com a separação da propriedade e do controle. No entanto, segundo La Porta et al (1999) a maioria das empresas grandes em todo o mundo são controladas por seus fundadores, ou por seus herdeiros e familiares. Heinz, Andrade e Barra (2001) argumentam que nem sempre todos os membros têm condições de controlar a riqueza em conjunto e, mesmo que isso ocorra, nem todos têm os mesmos objetivos para essa riqueza. Um estudo efetuado por esses autores em empresas familiares da América Latina aponta dois modelos de governança na empresa familiar: a governança "igualitária" e outra "caracterizada pela concentração de capital". A abordagem igualitária consiste em prever a participação de todos os membros da família, nos resultados da 
empresa e na herança, independente de talento e de sexo. Mesmo que alguns integrantes sequer desempenhem qualquer papel na empresa (HEINZ; ANDRADE; BARRA, 2001).

Nesses casos, a estrutura de governança deve contemplar um conselho de família responsável por: disseminar a cultura da família, definir as políticas familiares (dividendos, ações, permanência na empresa); eleger o Conselho de Administração que, preferencialmente, deve ser formado por profissionais de mercado e independentes. Caso o Conselho de Administração seja composto por integrantes da família, esses devem ser selecionados por profissional independente que seja capaz de indicar os candidatos que possuam as habilidades necessárias para assumir a administração dos negócios (HEINZ; ANDRADE; BARRA, 2001), (HAUSER, 2004).

A abordagem de concentração de capital tem como objetivo assegurar o poder da família ao longo do tempo através da concentração das ações no poder de apenas alguns membros da família (geralmente àqueles mais motivados e envolvidos com o negócio). Nessa abordagem o conselho de família é criado para resolver problemas familiares; promover valores comuns; esclarecer para as próximas gerações por que a gestão dos negócios se concentra nas mãos de poucos e estabelecer regras para a negociação de ações (HEINZ; ANDRADE; BARRA, 2001). A governança familiar funciona da mesma forma como qualquer outra forma de governo. Um bom sistema de governança é aquele que funciona de forma justa para os seus membros e se adapta bem à evolução das circunstâncias.

\section{Metodologia}

O desenvolvimento desta pesquisa se deu em nível exploratório, uma vez que o tema carece de uma visão geral sobre a produção científica sobre family office. O que permite desenvolver, esclarecer e modificar conceitos e idéias" a serem desenvolvidas em novos estudos.

O levantamento de evidencias foi desenvolvido, primeiramente, pela seleção das bases de dados a serem pesquisadas. A pesquisa foi efetuada através do portal EBSCOHost onde foram selecionadas as bases de dados: Academic Search Complete, Academic Search Premier, Business Source Complete e Regional Business News. O termo utilizado para pesquisa foi: Family Office, o qual foi digitado entre aspas para garantir o resultado para o significado do termo completo. $\mathrm{O}$ termo poderia ser encontrado: no corpo de texto, no titulo, no resumo e nas palavras chaves. Em seguida refinou-se a pesquisa solicitando os resultados que apresentassem o texto completo e revisados por especialistas. Essa busca produziu obter o resultado de 21 publicacoes. Complementarmente, foi efetuada pesquisa livre pela Internet. Os resultados estão apresentados no quadro abaixo e estão agrupados por periódico:

\begin{tabular}{|c|c|c|}
\hline Periódico & $\hat{A m b i t o}$ & Material \\
\hline CPA Journal & Internacional & 1 artigo \\
\hline Family Business Review & Internacional & 1 artigo \\
\hline Journal of Accountancy, & Internacional & 1 artigo \\
\hline Journal of Financial Planning, & Internacional & 1 artigo \\
\hline Journal of Sociology & Internacional & 1 artigo \\
\hline The Journal of Wealth Management & Internacional & 18 artigos \\
\hline McKinsey Quarterly & Internacional & 2 artigos \\
\hline Springer Science Business Media & Internacional & 2 artigos \\
\hline Trusts \& Trustees,April 2001;7:8 - 14. & Internacional & 5 artigos \\
\hline RACRE Revista de administração (2006) & Nacional & 1 artigo \\
\hline Revista de Administração e Negócios da Amazônia & Nacional & 1 artigo \\
\hline Revista de Negócios & Nacional & 1 artigo \\
\hline Revista jurídica FURB & Nacional & 1 artigo \\
\hline TCC Administração - UFRGS & Nacional & $1 \mathrm{TCC}$ \\
\hline Total & & 37 estudos \\
\hline
\end{tabular}

Quadro 4 - Publicações por periódico (elaborado pelas autoras) 
A partir da amostra dos 37 estudos, desenvolve-se a analise das publicações, classificando as mesmas, identificando as abordagens metodológicas utilizadas, analisando por autor (quantidade por artigo e número de publicações), por ano, por periódico e as obras mais citadas.

\section{Análise dos resultados}

A partir da pesquisa efetuada, foi possível perceber que o primeiro artigo sobre o tema foi publicado por Jon Carrol, em 2001, no The Journal of Wealth Managements. O objetivo do seu trabalho foi descrever as funções dos altos executivos e dos cargos administrativos de um family office. Porém, assim como as demais publicações daquele ano, trata-se de artigo opinativo, ou seja, não observa a metodologia científica para a obtenção dos resultados. Segue a classificação dos estudos por ano:

\begin{tabular}{|l|r|r|r|r|r|r|r|r|r|r|r|r|}
\hline Ano & 2001 & 2002 & 2003 & 2004 & 2005 & 2006 & 2007 & 2008 & 2009 & 2010 & 2011 & Total \\
\hline Nacional & - & - & - & - & - & 1 & 1 & 1 & 1 & - & 1 & 5 \\
\hline Internacional & 3 & 1 & 1 & 3 & 3 & 3 & 4 & 5 & 3 & 5 & 1 & 32 \\
\hline
\end{tabular}

Quadro 5 - Publicações por ano (elaborado pelas autoras)

Pode-se observar que o ano de 2008 foi o ano que mais apresenta publicações sobre o tema em questão, apresentando o total de 6 publicações cada. Percebe-se que não há uma evolução expressiva nas pesquisas na última década. Pode-se verificar um interesse tímido a partir de 2008. No Brasil, o assunto começa a ser abordado em 2006. No entanto, os estudos não abordam exatamente o tema family office, e quando o abordam, o fazem com superficialidade. Os autores brasileiros pesquisam a empresa familiar e suas questões de governança, como, por exemplo, o trabalho de Escuder (2006) que teve por objetivo estudar os instrumentos de controle e gestão utilizados na redução ou eliminação dos conflitos societários e na avaliação da importância do conselho fiscal na estrutura das organizações como instrumento de controle e apoio à Assembléia de Acionistas. $\mathrm{O}$ artigo abordou o tema de maneira conceitual e teórica.

Vlizumoto e Machado Filho (2007) abordaram as práticas de governança corporativa em empresas familiares de capital fechado, através de um estudo de caso junto ao Grupo Orsa. Os autores apresentam, no referencial bibliográfico, os conceitos de governança corporativa e os aspectos que cercam a gestão de empresas familiares. A metodologia constou de entrevistas presenciais com os dirigentes da empresa, bem como com seus consultores.

Franke (2008) analisou as possibilidades jurídicas e a viabilidade econômica na constituição de empresas administradoras de bens próprios. Foram apresentadas três estruturas jurídicas possíveis, sendo elas: sociedade limitada, sociedade anônima e offshore company. A autora abordou ainda os aspectos tributários incidentes sobre cada uma das estruturas.

O único estudo brasileiro encontrado que aborda especificamente o tema family office foi o trabalho de conclusão para o curso de pós-graduação em Administração na Universidade Federal do Rio Grande do Sul escrito por Aquino (2009). O objetivo do trabalho, segundo o autor, foi o de definir family office e demonstrar o seu funcionamento no Brasil. O trabalho fundamenta-se em informações coletadas da internet, mas especificamente de sites de instituições que oferecem esse tipo de serviço, bem como de reportagens que abordam o assunto. $\mathrm{O}$ autor apresenta alguns dados interessantes, tais como: Em 2006, estimativas apresentadas pelo The Boston Consulting Group, apontavam que o Brasil possuía 130 mil milionários e em 2007 essa estimativa havia aumentado para 190 mil. O autor informa ainda (sem mencionar a fonte) que o Brasil possuía, na época, pelo menos 15 family offices com patrimônio entre R 200 a R \$ 250 milhões de reais. Cita alguns family offices brasileiros, tais como Grupo Gerdau, Natura, Ermírio de Moraes, Roberto Marinho (Grupo RBS). 
Dias e Nenevê (2011) também estudaram a gestão familiar. O foco do trabalho dos autores foi a trajetória da profissionalização e a sucessão nas empresas familiares de São Bento do Sul, Santa Catarina. Os dados foram coletados mediante entrevistas semi-estruturadas, visando conhecer o modelo de gestão adotado por cinco empresas situadas na referida região. Os autores discorrem sobre as peculiaridades das empresas familiares, bem sobre como as instâncias de governança requeridas nesse estilo de empresa, embasados por bibliografia nacional. A análise dos resultados indica uma forte presença de um modelo conservador de gestão nas empresas analisadas. Esse modelo se caracteriza, principalmente, pela centralização das decisões na pessoa do fundador. Observou-se também a forte presença de membros da família ocupando cargos de gestão na empresa (mesmo sem as competências necessárias para tanto). Das cinco empresas entrevistadas, apenas uma possui uma estrutura administrativa totalmente profissional, ou seja, os cargos de gestão não são ocupados por parentes. Todas as empresas possuem, em alguma medida, normas com relação a sucessão e um conselho de administração (formado parte por familiares e parte por profissionais de mercado).

A partir da análise por periódico, pode-se observar que o The Journal of Wealth Management, destacou-se com a publicação de $49 \%$ da produção sobre family office, tendo, inclusive, conforme mencionado anteriormente, publicado o primeiro artigo sobre o tema.

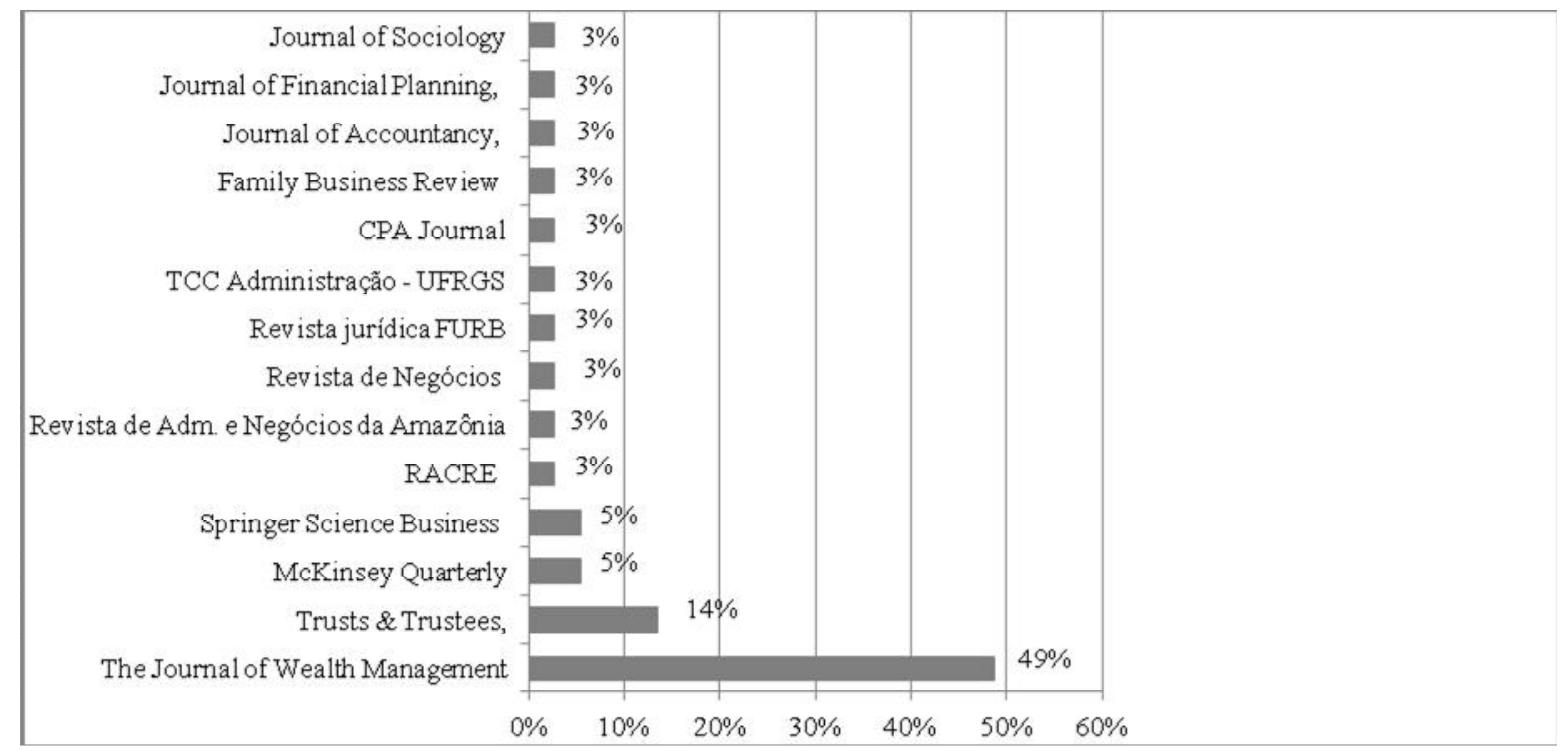

Figura 1 - Publicações por periódico (elaborado pelas autoras)

A análise quanto a abordagem metodológica centrou-se na classificação quanto a coleta e o tratamento dos dados, observando os seguintes delineamentos:

a) Pesquisa documental: Trabalhos cujo resultado foi obtido através da análise de documentos (relatórios de pesquisa, relatórios de empresas, tabelas estatísticas).

b) Estudo de caso: Trabalhos cujo resultado foi obtido mediante a análise aprofundada de um ou de poucos itens. Foram incluídos nessa classificação os casos hipotéticos citados pelos autores para o aprofundamento do assunto.

c) Survey: Trabalhos cujos dados foram coletados mediante aplicação de questionários objetivos.

d) Entrevista: Trabalhos cujos dados foram coletados mediante a interlocução entre o entrevistador e o entrevistado.

e) Econométricos: Trabalhos cujos resultados foram obtidos mediante a utilização da econometria. 
f) Opinativos: Nesses casos não há observância do método científico na coleta e no tratamento dos dados.

A partir da análise quanto à abordagem metodológica, contata-se um predomínio de artigos opinativos, com $76 \%$ das publicações revisadas. Esses trabalhos não observam o rigor científico na sua elaboração, entretanto, seu conteúdo é pertinente ao tema tratado. Os autores não são pesquisadores acadêmicos, mas sim consultores de mercado que traduzem sua experiência nos referidos trabalhos. Esse fato reforça o argumento de que o family office é um assunto pouco explorado pela academia e por essa razão o presente artigo representa uma contribuição nesse sentido.

Os poucos trabalhos empíricos não tratam especificamente de family office. Os estudos de casos prevalecem na análise da governança corporativa em empresa familiar, nessas situações o family office é abordado como um dos componentes da governança familiar. Os estudos econométricos também não são focados em family office. O estudo efetuado por Evans (2004) envolveu a análise da relação entre o perfil de risco informado pelo investidor e o perfil de risco de suas carteiras de investimento. Nesse estudo, o autor conclui que não há correlação entre essas duas variáveis, mas quando os investimentos são efetuados por family offices identifica-se uma pequena correlação. O outro estudo econométrico busca analisar aspectos de governança corporativa através da análise de 56 IPOs italianos emitidos entre 1999 e 2005. As hipóteses buscam testar a relação entre governança corporativa, propriedade familiar e desempenho. A survey aplicada por Braun e Uhlaner (2010) objetivou explorar a relação entre práticas de governança familiar e desempenho financeiro dos negócios da família. As autoras aplicaram questionários a um universo de 64 empresas familiares em 18 países. Por fim, a entrevista conduzida por Gilding (2005) teve por objetivo argumentar que as relações familiares são uma' influência duradoura em relação à acumulação, sucessão e herança. Ele entrevistou 43 ricos australianos que constavam na 'Rich 200' publicada pela Business Review Weekly (BRW) na Austrália.

A autora que mais escreve sobre o tema é Lisa Gray, CEO da Graymatter Strategies L.L.C em Memphis, TN, com 70\% das publicações encontradas. Seguida por Sara Hamilton, que também é profissional de mercado, CEO e fundadora da Family Office Exchange em Chicago, com 14\% das publicações encontras nos periódicos revisados. Peter Elstrodt Hein é líder do escritório da McKinsey Quarterly no Mercosul e Charles A e Lowenhaupt é executivo membro do Lowenhaupt \& Chasnol T,LLC, em St. Louis, MO. Cada um deles obtem 5\% das publicações encontradas. Como já comentado anteriormente, todos tem em comum o fato de serem profissionais de mercado.

Quanto ao número de autores por artigo, observa-se que há predominância de autoria isolada. Esse fato poderia ser explicado pelo fato de que a maioria dos autores não está relacionada à academia, mas sim ao mercado empresarial, podendo, em alguns casos, prestar serviços para empresas concorrentes. Os Autores mais citados, tanto a nível internacional quanto a nível nacional são apresentados na tabela abaixo:

\begin{tabular}{|l|l|l|l|}
\hline Artigos internacionais & $n^{\circ}$ citaç̃̃es & Artigos Nacionais & $n^{\circ}$ citações \\
\hline Brunel, Jean L.P. & 7 & Lodi, J. B. A & 4 \\
\hline Gray, Lisa & 7 & Bulgarelli, W. & 3 \\
\hline Uhlaner, L. M. \& Berent, M. M. & 7 & Andrade, A. Rosseti, J.P. & 2 \\
\hline Reichenstein, William & 5 & Babic, V. & 2 \\
\hline Ward, J. & 4 & Bernhoeft, R. \& Gallo, M. & 2 \\
\hline Dyer Jr.,W. G. & 4 & Bernhoeft, R. & 2 \\
\hline Berle, A. and G. Means & 3 & Donelley, R.G. & 2 \\
\hline Chua, J. H.,Chrisman, J. J.,\& Sharma, P. & 3 & IBGC & 2 \\
\hline FOX Research. & 3 & Leone, N.M.de C.P.G. & 2 \\
\hline
\end{tabular}




\begin{tabular}{|l|l|l|l|}
\hline Gilding, M. & 3 & Oliveira, D. de P. R. de. & 2 \\
\hline Handler, W. C. & 3 & & \\
\hline Jensen, M. C., \& Meckling, W. H. & 3 & & \\
\hline Kahneman, Daniel & 3 & & \\
\hline Neubauer, F. and A.G. Lank & 3 & & \\
\hline Schultze, W. S., Lubatkin, M. H., \& Dino, R. H. & 3 & & \\
\hline Villalonga A, B. Amit, R. & 3 & & \\
\hline
\end{tabular}

Quadro 6: Autores mais citados em publicações - internacionais e nacionais (elaborado pelas autoras)

Dos trabalhos abrangidos pela amostra do presente artigo, as obras mais citadas a nível internacional são as de autoria de Lisa Gray, Jeam L.P. Brunel e Uhlaner, L. M. \& Berent, M. M., todos com 7 (sete) citações cada. Desses autores, Lisa Gray é a autora que escreve especificamente sobre family office. Jean P. Brunel aborda a gestão de riqueza e Uhlaner, L. M. $\&$ Berent, M. M., as perspectivas geracionais.

No contexto nacional, o trabalho do professor João Bosco Lodi é o que mais se destaca com 4 (quatro) citações. Seu trabalho aborda o papel do conselho de administração na estrutura de governança corporativa.

\begin{tabular}{|c|c|c|}
\hline Autor & Artigo & $\begin{array}{l}n^{\circ} \text { de } \\
\text { citações }\end{array}$ \\
\hline Berle, A. and G. Means & The modern corporation and private property & 3 \\
\hline $\begin{array}{l}\text { Jensen, M. C., \& Meckling, } \\
\text { W.H. }\end{array}$ & $\begin{array}{l}\text { Theory of the firm: managerial behavior, agency costs and ownership } \\
\text { structure }\end{array}$ & 3 \\
\hline Villalonga, B. Amit, R. & How do family ownership, control, and management affect firm & 3 \\
\hline Bulgarelli, W. & O conselho fiscal nas companhias brasileiras & 3 \\
\hline $\begin{array}{l}\text { Astrachan, J. H., Klein, S. B., } \\
\text { \& Smyrnios, K. X. }\end{array}$ & $\begin{array}{l}\text { The F-PEC scale of family influence: construction, validation, and } \\
\text { further implication for theory. }\end{array}$ & 2 \\
\hline Brunel, Jean L.P. & $\begin{array}{l}\text { Revisiting the asset allocation challenge though a behavioral finance } \\
\text { lens. }\end{array}$ & 2 \\
\hline $\begin{array}{l}\text { Day, George S., and Paul J.H. } \\
\text { Shoemaker }\end{array}$ & Scanning the Periphery & 2 \\
\hline Dyer. W. G. & Examining the "family effect" on firm performance & 2 \\
\hline Fama, E., \& Jensen, M. & Separation of ownership and control & 2 \\
\hline Gray, Lisa & The new family office innovative strategies for consulting to the affluent & 2 \\
\hline Gray, Lisa & $\begin{array}{l}\text { Generational perspectives and their effects on goalbased } \\
\text { Allocation }\end{array}$ & 2 \\
\hline Mustakallio, M. et al & $\begin{array}{l}\text { Relational and contractual governance in family firms: } \\
\text { effects on strategic decision making }\end{array}$ & 2 \\
\hline Neubauer, F. and A.G. Lank & The family business: its governance for sustainability & 2 \\
\hline Shleifer, A., \& Vishny, R. & A survey of corporate governance & 2 \\
\hline Andrade A;Rossetti, J.P. & Governança corporativa - fundamentos, desenvolvimento e tendências & 2 \\
\hline Bernhoeft, R. \& Gallo, M. & Governança na empresa familiar: poder, gestão e sucessão & 2 \\
\hline Bernhoeft, R & $\begin{array}{l}\text { Empresa familiar: sucessão profissionalizada ou sobrevivência } \\
\text { comprometida }\end{array}$ & 2 \\
\hline Lodi, J.B. & $\begin{array}{l}\text { Governança corporativa: o governo da empresa e o conselho de } \\
\text { administração }\end{array}$ & 2 \\
\hline Oliveira, D. de P. R. de. & $\begin{array}{l}\text { Empresa familiar: como fortalecer o empreendimento e otimizar o } \\
\text { processo sucessório }\end{array}$ & 2 \\
\hline
\end{tabular}

Quadro 7 - Obras mais citadas (elaborado pelas autoras)

As obras mais citadas referem-se aos clássicos sobre governança corporativa escritos por Berle e Means e Jensen e Meckling. Ambos se destacam com 3 citações cada. 


\section{Considerações finais}

Os family offices são estruturas profissionais que prestam todo tipo de serviços a famílias ricas. Eles surgiram, inicialmente, para auxiliar as famílias a gerenciarem o produto gerado pelo negócio: a riqueza. Atualmente, os family offices podem prestar qualquer tipo de serviço que uma família possa desejar. Os estudos enfatizam os seguintes: planejamento sucessório, aspectos tributários, gestão e alocação de ativos, treinamento e serviços de concierge.

Essas estruturas existem, na prática, há bastante tempo, no entanto, são pouco exploradas pela academia, no que diz respeito à produção científica. Por essa razão, o presente estudo teve por objetivo identificar o estado da arte sobre family Office. Para tanto, foi realizada pesquisa bibliográfica a partir da análise da base de dados EBESCOhost e também mediante pesquisa livre pela internet. A partir dessa pesquisa, obtivemos acesso a 37 (trinta e sete) trabalhos que faziam referência a family office. Essa amostra compôs o corpo de análise. A partir da análise dessa amostra verificou-se que:

(a) - o periódico que mais publica sobre o assunto é The Journal of Wealth Managements com 49\% (quarenta e nove por cento) das publicações, seguido do Journal of Accountancy com 14\% (quatorze por cento) das publicações.

(b) O tema vem sendo estudado desde 2001, porém há poucas publicações por ano. O ano que se destaca em relação à quantidade de publicações é o ano de 2008 com o total de seis publicações;

(c) Do total dos 37 (trinta e sete) artigos, apenas 5 (cinco) são brasileiros.

Diante dessa análise preliminar, levando em consideração o número de trabalhos encontrados (37) em um período de 10 anos (2001 a 2011), pode-se concluir que a pesquisa sobre family office é ainda incipiente. Em nível nacional, a partir dos dados coletados, pode-se afirmar que a pesquisa científica é nula, uma vez que, dos 5 (cinco) artigos apenas 1 (um) trata especificamente do assunto, porém sem observar o rigor científico.

Os resultados obtidos do estudo bibliométrico podem ser sintetizados da seguinte forma:

(a) Predomínio de trabalhos opinativos, abrangendo $70 \%$ da amostra;

(b) A autora Lisa Gray lidera a lista de autores que mais escrevem sobre o assunto;

(c) Predomínio de autores profissionais de mercado e não acadêmicos.

Sendo assim, evidenciou-se que não há muitos estudos acadêmicos, nem a nível internacional e nem a nível nacional, visto que $70 \%$ do total da produção não observa o rigor científico, sendo classificados como artigos opinativos. Dessa forma, foi possível identificar um espaço amplo a ser explorado no que diz respeito a execução de pesquisas empíricas que observem o rigor científico sobre o assunto.

\section{Referências}

AQUINO, R. V. Family Office. 2009. 40f. Trabalho de Conclusão de Curso de Especialização (Especialização em Administração) - Programa de Pós-Graduação em Administração, Universidade Federal do Rio Grande do Sul, Porto Alegre, RS, 2009. Disponível em http://www.lume.ufrgs.br/bitstream/handle/10183/26261/000748791.pdf? sequence=1. Acesso em: 5 julho 2011.

BERLE, A., MEANS, G. The modern corporation and private property. New York: Harcourt, 1932.

BRUNEL, J. L. P.; GRAY, L. P. Integrating family dynamics and governance in strategic asset allocation. The Journal of Wealth Management. v. 8, n. 3, p. 39-49, 2005. 
CARROL, J. The functions of a family office. The Journal of Wealth Management. v.4, n. 2, p. 23-27, 2001.

CHITTOOR, R.; DAS, R. Professionalization of management and succession performance-A vital linkage. Family Business Review. v. 20, n. 1, p. 65-79, mar. 2007.

ESCUDER, S. L. Empresa familiar e os mecanismos e instrumentos facilitadores na gestão de conflitos societários. Revista de Administração da UNIMEP. v. 4, n. 3. p. 80, set. 2007.

EVANS, L. J. Wealthy investor attitudes, Expectations, and Behaviors toward risk and return. Journal of Wealth Management. v. 7, n. 1, p. 12-18, 2004.

FRANKE, L. P. Possibilidades jurídicas e viabilidade econômica na constituição de empresas administradoras de bens próprios. Revista jurídica FURB. v. 12, p. 95 - 111, 2008.

GARNHAM, C. The Growth of the family office and the challenge for the Future. Trusts \& Trustees. v. 7, p. $8-14,2001$.

GILDING, M. Families and fortunes: Accumulation, management succession and inheritance in wealthy families. Journal of Sociology., v. 41, n. 1, p. 29-45, mar. 2005.

GILLAN, S. L. Recent developments in corporate governance: An overview. Journal of corporate finance. v. 12, 2006.

GRAY, L. Generational perspectives and their effects on goal-based allocation. The Journal of Wealth Management. v. 9, n. 1, p. 7-17, 2006.

GRAY, L. How family dynamics influence the structure of the family office. The Journal of Wealth Management. v. 8, n. 2, p. 9-17, 2005.

GRAY, L. The three forms of governance: A new approach to family wealth transfer and asset protection, part I. The Journal of Wealth Management. v. 10, n. 2, p. 10-19, 2007.

HAMILTON, S.; GODFREY, J. Preparing the next generation for the responsibilities of ownership. The Journal of Wealth Management. v. 10, n. 3, p. 8-16, 2007.

HAMILTON, S. Window on the future: Observations on the future of the family wealth marketplace. The Journal of Wealth Management. v. 9, n. 4, p. 7-12, 2007.

HAUSER, B. R. Family governance in 2004. The Journal of Wealth Management. v. 7, n. 1, p. 48-55, 2004.

HEINZ, P. E. Keeping the family in business. McKinsey Quarterly. v.4, p. 94-103, 2003.

HEINZ, P. E.; ANDRADE, L. F.; BARRA, J. M. All in the família. McKinsey Quarterly. v.4, p. 81-89, 2001.

IBGC - Instituto brasileiro de governança corporativa. Código das melhores práticas de governança corporativa. Disponível em: <http://www.ibgc.org.br $>$. Acesso em: 3 de maio de 2011.

ISDALE, M. H. Strategies for simplifying and increasing the effectiveness of wealth management. The Journal of Wealth Management. v. 9, n. 2, p. 14-18, 2006.

JENSEN, M.; MECKLING, W. Theory of the firm: managerial behavior, agency costs and ownership structure. The Journal of financial economics, v. 3, 1976. 
LA PORTA, R.; SHLEIFER, A., LOPEZ-DE-SILANES, F. Corporate ownership around the world. Journal of finance, v. 54, 1999.

LOWENHAUPT, C. A. Freedom from wealth. The Journal of Wealth Management. v. 10, N. 4, p. 17-23, 2008.

LOWENHAUPT, C. A. Freedom from wealth and the contemporary global family: A new vision for family wealth management. Journal of Wealth Management. v. 11, n. 3, p. 21-29, 2008.

MASLINSKI , M. The family office: adapting to modern needs in a changing world. Trusts $\boldsymbol{\&}$ Trustees. v. 12, n. 6, p. 27-29, 2006.

MIZUMOTO, F. M.; MACHADO FILHO, C. P. Práticas de governança corporativa em empresa familiar de capital fechado: um estudo de caso. Revista de Negócios. v. 12, n. 2, p. 3-17, 2007.

MONTFORD, C. Family office type investment management services for trustees and high net worth investors. Trusts \& Trustees. v. 10, n. 3, p. 27 - 28, 2004.

MORCK, R. K. (editor). A history of corporate governance around the world. Chicago: National Bureau of Economic Research - NBER, 2005.

NEWTON, C. Adopting the Family Office. Journal of Financial Planning. v. 15, n. 6, p. 66-74, 2002.

SHLEIFER, A.; VISHNY, R. A survey of corporate governance. Journal of Finance. v. 52, n. 2, p. 737-775, 1997. 